in total number of both sexual partners overall and with sexual partners who did not use condoms. In contrast, HIV status, education, number of immediate family members and levels of alcohol consumption were non-significant factors for both regression analyses.

Conclusion Results suggest that female sex workers' romantic partners act as more than sources of possible HIV infection; rather, romantic partners appear to have an important positive impact on health. We discuss this finding in light of possible harm-reduction programmes focusing on female sex workers and their romantic partners.

\section{P3.171 MALE SPOUSE PERPETRATED PSYCHOLOGICAL AND SEXUAL ABUSE AMONG PREGNANT WOMEN IN NAIROBI, KENYA}

${ }^{1}$ Njeri Mbugua, ${ }^{2}$ Elizabeth Ann Bukusi, ${ }^{3}$ Elizabeth Ngugi, ${ }^{3}$ James Mwaura, ${ }^{3}$ David Nguti. ${ }^{1}$ Kenya Medical Research Institute/Nairobi University/Kenyatta Hospital/Kenya Women With HIVIAIDS, Nairobi, Kenya; ${ }^{2}$ Kenya Medical Research Institute/Nairobi University, Nairobi, Kenya; ${ }^{3}$ Nairobi University, Nairobi, Kenya

10.1136/sextrans-2017-053264.406

Introduction The purpose of this comparative retrospective study was to evaluate the nature of male spouseperpetrated gender based violence (GBV) during pregnancy. The objective was to establish whether diagnosis of HIV infection during pregnancy mitigates or exacerbates male spouse perpetrated psychological and sexual abuse during pregnancy.

Methods Case group comprising 96 HIV infected pregnant women, and comparison group (96 uninfected), all in their third trimester of pregnancy were interviewed upon consenting. A modified Conflict Tactics Scale 2 was administered to compare the two groups in terms of psychological aggression and sexual coercion.

Results Results indicated prevalence and severity of male spouse perpetrated abuse to be higher for case group than comparison group across both psychological aggression and sexual coercion subscales. The odds of male spouse perpetrated violence was 6.64fold higher in HIV positive pregnant women compared to HIV negative pregnant women $(\mathrm{OR}=6.64,95 \% \mathrm{CI}$ 1.56-28.27, $\mathrm{p}=0.010$ ). Thus, diagnosis of pregnancy and absence of HIV infection was associated with mitigated occurrence and severity of male spouse perpetrated abuse, while diagnosis of HIV infection during pregnancy exacerbated the same.

Conclusion The investigator recommends immediate sensitisation of health and social workers attending to pregnant women on the escalative effect of HIV positive diagnosis on male-spouse perpetrated violence. Intensive couple counselling and follow up care need to be specially designed and implemented for such couple whether they are concordant positive or discordant.

\section{P3.172 BACTERIAL VAGINOSIS: RISK FACTORS AMONG KENYAN WOMEN AND THEIR MALE PARTNERS}

${ }^{1}$ Njeri Mbugua, ${ }^{2}$ Elizabeth Ann Bukusi, ${ }^{3}$ Waiyaki Peter. ${ }^{1}$ Kenya Medical Research Institute/ Nairobi University/Kenyatta Hospital/Kenya Women With HIVIAIDS, Nairobi, Kenya; ${ }^{2}$ Kenya Medical Research Institute/Nairobi University, Nairobi, Kenya; ${ }^{3}$ Nairobi University/Kenya Medical Research Institute, Nairobi, Kenya

10.1136/sextrans-2017-053264.407
Introduction To simultaneously examine associations of bacterial vaginosis (BV) with potential risk factors in both the female and her male partner.

Methods We recruited women 18-45 years of age and their male partners from clinics in Nairobi, Kenya. All underwent face-to-face standardised interview physical examination, human immunodeficiency virus (HIV) -1 and syphilis serologic testing, endocervical cultures for Neisseria gonorrhoeae, and vaginal swabs for diagnosis of BV by Gram stain and trichomoniasis by culture.

Results Of 219 women, 97 (44\%) had BV. BV was significantly associated by univariate analyses with women's own risk factors (young age, being unmarried, early sexual debut, more than 1 sexual partner, lifetime, rectal sex, trichomoniasis, HIV infection, and by principal components analysis, with low socioeconomic status [SES]) and also with male partners' characteristics (HIV infection, and by principal components analysis, low SES, and poor hygiene). In multivariate analysis including risk factors from both genders, the odds of having BV was 5.7 times higher if either partner was HIV seropositive, 13.2 times higher if the female had trichomoniasis, 2.5 times higher if the female had more than 1 sex partner ever, and decreased with increasing age of the female.

Conclusion In this population, characteristics of males and of females were independently associated with BV. Close association of male hygiene and male HIV status precluded distinguishing the influence of male hygiene on partner's BV.

\section{P3.173 TRIPLE-ANTIRETROVIRAL PROPHYLAXIS TO PREVENT MOTHER-TO-CHILD HIV TRANSMISSION THROUGH BREASTFEEDING-THE KISUMU BREASTFEEDING STUDY, KENYA: A CLINICAL TRIAL}

${ }^{1}$ Njeri Mbugua, ${ }^{2}$ Elizabeth Ann Bukusi, ${ }^{3}$ Oyugi Julius Otieno. ${ }^{1}$ Kenya Medical Research Institute/Nairobi University/Kenyatta Hospital/Kenya Women With HIVIAIDS, Nairobi, Kenya; ${ }^{2}$ Kenya Medical Research Institute/Nairobi University, Nairobi, Kenya; ${ }^{3}$ Nairobi University, Nairobi, Kenya

\subsection{6/sextrans-2017-053264.408}

Introduction Effective strategies are needed for the prevention of mother-to-child HIV transmission (PMTCT) in resource-limited settings. The Kisumu Breastfeeding Study was a single-arm open label trial conducted between July 2003 and February 2009. The overall aim was to investigate whether a maternal triple-antiretroviral regimen that was designed to maximally suppress viral load in late pregnancy and the first 6 months of lactation was a safe, well-tolerated, and effective PMTCT intervention.

Methods and findings HIV-infected pregnant women took zidovudine, lamivudine, and either nevirapine or nelfinavir from 34-36 weeks' gestation to 6 months post partum. Infants received single-dose nevirapine at birth. Using Kaplan-Meier methods we estimated HIV-transmission and death rates from delivery to 24 months. We compared HIV-transmission rates among subgroups defined by maternal risk factors, including baseline CD4 cell count and viral load. Among 487 live-born, singleton, or first-born infants, cumulative HIV-transmission rates at birth, 6 weeks, and 6,12 , and 24 mo were $2.5 \%$, $4.2 \%, 5.0 \%, 5.7 \%$, and $7.0 \%$, respectively. The 24-mo HIVtransmission rates stratified by baseline maternal CD4 cell count $<500$ and $\geq 500$ cells $/ \mathrm{mm}^{3}$ were $8.4 \%$ (95\% confidence interval $[\mathrm{CI}] 5.8 \%-12.0 \%)$ and $4.1 \%$ (1.8\%-8.8\%), respectively $(p=-0.06)$; the corresponding rates stratified by baseline maternal viral load $<10000$ and $\geq 10000$ copies/ml were 
$3.0 \% \quad(1.1 \%-7.8 \%)$ and $8.7 \% \quad(6.1 \%-12.3 \%)$, respectively $(p=0.01)$. None of the 12 maternal and 51 infant deaths (including two second-born infants) were attributed to antiretrovirals. The cumulative HIV-transmission or death rate at 24 mo was $15.7 \%$ (95\% CI 12.7\%-19.4\%).

Conclusion This trial shows that a maternal triple-antiretroviral regimen from late pregnancy through 6 months of breastfeeding for PMTCT is safe and feasible in a resource-limited setting. These findings are consistent with those from other trials using maternal triple-antiretroviral regimens during breastfeeding in comparable settings.

\section{P3.174 PREVENTION OF HUMAN IMMUNODEFICIENCY VIRUS BREASTMILK TRANSMISSION WITH COPPER OXIDE: PROOF-OF-CONCEPT STUDY}

${ }^{1}$ Njeri Mbugua, ${ }^{2}$ Oyugi Julius Otieno. 'Kenya Medical Research Institute/Nairobi University/ Kenyatta Hospital/Kenya Women With HIVIAIDS, Nairobi, Kenya; ${ }^{2}$ Nairobi University, Kenya, Kenya

\subsection{6/sextrans-2017-053264.409}

Introduction Human immunodeficiency virus type 1 (HIV-1) transmission through breastmilk is the chief modality through which HIV-1 is transmitted from HIV-1-infected mothers to their babies in developing countries, where alternative feeding options lack practical feasibility. The development of an approach to inactivate the HIV-1 virions ingested by an infant on a daily basis through breastmilk is thus of critical importance.

Methods Copper has potent virucidal properties. Stoichiometric concentrations of copper ions inactivate the HIV-1 protease, which is essential for viral replication. Cell-free and cell-associated HIV-1 infectivity is inhibited when the virus is exposed to copper oxide in a dose-dependent manner. Passage of high titers of a wide range of HIV-1 isolates, spiked in culture medium, through filters containing copper oxide powder resulted in their deactivation.

Results In the current study, we demonstrate that the infectivity of three different HIV-1 isolates, spiked in breastmilk obtained from HIV-1-seronegative donors, or of wild-type isolates found in breastmilk obtained from HIV-1-seropositive donors, is drastically reduced (>98\%) when exposed to copper oxide.

Conclusion This study is proof of concept that copper oxide is efficacious against HIV-1 found in breastmilk and serves as the basis for further research aimed at determining the possible effects that copper may have on the nutritional and antiinfective properties of breastmilk. Furthermore, this supports the continuing study of the feasibility of developing a filtering device, such as an "at-the-breast" disposable shield that can be used discreetly and safely by HIV-1-infected mothers during breastfeeding.

\section{P3.175 EARLY DEVELOPMENT OF BROADLY NEUTRALISING ANTIBODIES IN HIV-1-INFECTED INFANTS}

${ }^{1}$ Njeri Mbugua, ${ }^{2}$ Elizabeth Ann Bukusi, ${ }^{3}$ Asunta Wagura, ${ }^{4}$ Elizabeth Ngugi. ${ }^{1}$ Kenya Medical Research Institute/Nairobi University/Kenyatta Hospital/Kenya Women With HIV/AIDS, Nairobi, Kenya; ${ }^{2}$ Kenya Medical Research Institute/Nairobi University, Nairobi, Kenya; ${ }^{3}$ Kenya Network of Women Living With Hiv/Aids, Nairobi, Kenya; ${ }^{4}$ Nairobi University, Nairobi, Kenya
Introduction We evaluated predictors of consistent condom use among female sex workers (FSWs), a core group for controlling the spread of HIV.

Methods In an analysis of data collected in 2004-2005 from 140 Kenyan FSWs who completed questionnaires administered during a baseline study visit and three bimonthly follow-up visits, we used a case-crossover design to identify predictors of consistent condom use during all coital acts in the preceding 2 weeks, overall and by partner type.

Results Participants $(n=140)$ completed the baseline visit and 390 bimonthly follow-up visits. Alcohol use during sex was negatively associated with consistent condom use with helping partners (defined as regular sex partners to whom the woman could go for help or support if needed) (adjusted odds ratio [AOR], 2.6, 95\% confidence interval [CI] 1.0-6.5) but not associated with condom use with other partners. Coital frequency was associated with condom use with other partners only. Women who reported 1-5 (AOR 11.0, 95\% CI 4.328.3) or 6-9 recent coital acts (AOR 3.8, 95\% CI 1.7-8.8) with other partners were more likely to report consistent condom use with those partners than were women who reported $\geq 10$ acts. Having a recent partner delay payment was inversely associated with consistent condom use with helping, other, or all partners.

Conclusion Correlates of consistent condom use differed by partner type. By using a case-crossover design, we were able to identify potentially modifiable factors associated with consistent condom use by FSWs who used condoms consistently with a given partner type during some periods but not others.

\section{P3.176 SERO-PREVALENCE OF SYPHILIS AMONG FEMALE SEX WORKERS IN MOROCCO}

${ }^{1} \mathrm{H}$ Oukouchoud, ${ }^{1} \mathrm{C}$ Ouanaim, ${ }^{1} \mathrm{~B}$ Bellaji, 'A Hançali, ${ }^{1} \mathrm{~S}$ Jennane, ${ }^{2} \mathrm{~A}$ Bennani, ${ }^{2} \mathrm{~A}$ Latifi, ${ }^{1} \mathrm{R}$ Charof. ${ }^{1}$ National Institute of Hygiene Rabat, Rabat, Morocco; ${ }^{2}$ National AIDS Program Ministry of Health, Rabat, Morocco

\subsection{6/sextrans-2017-053264.411}

Introduction Syphilis is a common but curable sexually transmitted infection (STI). Nevertheless the limited access to medical care among Female Sex Workers (FSWs) can reduce individual treatment, thereby indirectly facilitating transmission within the population. There is increased risk of HIV acquisition and transmission of HIV because of ulcerative syphilis. This study aimed to provide, for the first time using response driven sampling (RDS), baseline information on the prevalence and treatment rate of syphilis among FSWs in Morocco.

Methods This study was conducted from December 2011 to January 2012 in four Moroccan regions: Agadir, Rabat, Fes and Tangier. A total of 1447 FSWs participants were recruited using RDS. All participants completed an anonymous questionnaire on sex behavioural information and were tested for syphilis by using a combination of two tests; a non treponemal test (venereal diseases research laboratory VDRL) and treponemal test (treponema pallidum haemmaglutination assay: TPHA).

Results Among1447 FSWs (17.68\%) were reactive for syphilis with $21.4 \%$ in Agadir, $18.8 \%$ in Fes, $13.9 \%$ in Rabat and $13.3 \%$ in Tangier. Only $25.54 \%$ of FSWs reported being 
tested for HIV in the past 12 months and 4\% ever been tested for HIV, 50.25\% reported using a condom at last transactional sex.

Conclusion The prevalence of syphilis was high among female sex workers and majority of them were treated for syphilis. Achieving good treatment coverage therefore will help not only to reduce syphilis incidence but also HIV disease burden in the high risk population and general population since the relationship between syphilis and HIV is well established. This study has shown syphilis continues to be highly prevalent among female sex workers and targeted intervention programs need to focus on curable STI like syphilis.

\section{P3.177 IDENTIFICATION OF SUPEROXOL NEGATIVE NEISSERIA GONORRHOEAE ISOLATES OVER 25 YEARS IN ARGENTINA}

'Oviedo Claudia, 'Gonzalez Melisa, ${ }^{1}$ Gianecini Ricardo, ${ }^{1}$ Cristaldo Paula, ${ }^{2}$ Gassp-Ar, ${ }^{1}$ Galarza Patricia. 'Instituto Nacional de Enfermedades Infecciosas (INEI)-Anlis, Ciudad Autónoma de Buenos Aires, Argentina; ${ }^{2}$ Gonococcal Antimicrobial Susceptibility Surveillance Program-Argentina (GASSP-AR), Ciudad Autónoma de Buenos Aires, Argentina

\subsection{6/sextrans-2017-053264.412}

Introduction Isolation of Neisserias and other related microorganisms from genital and extra genital sites require the identification of clinically important isolates and represent a challenge in low-complexity clinical microbiology laboratories. A presumptive Neisseria gonorrhoeae $(\mathrm{Ng})$ identification includes the Superoxol test (Spx) among others. $\mathrm{Ng}$ produces levels of catalase activity that are stronger than others Neisseria spp. Spx is a simple and inexpensive test that uses $30 \%$ hydrogen peroxide as a reagent. Superoxol reaction with $\mathrm{Ng}$ is typically "explosive" compared to weaker reactions of other species. The objective is to report the finding of N. gonorrhoeae superoxol test negative over 25 years in Argentina.

Methods We studied 8047 isolates derived from the GASPPAR for susceptibility studies between 1990 and 2015. Presumptive identification of isolates was made on the colonies that developed in selective medium. All isolates were confirmed by carbohydrate utilisation and/or coagglutination with monoclonal antibodies and/or mass spectrophotometry (Bruker Daltonik). For Spx a positive result was defined as abundant production of bubbles occurring within two to three seconds of starting emulsification. A negative reaction was defined by weak or delayed bubbling after three seconds. Pulsed field gel electrophoresis (PFGE) was carried out using NheI enzyme.

Results Out of the total isolates studied, 99.9\% (8038/8047) showed a positive Spx, however 9 isolates were Spx negative. These isolates came from 5 different regions of the country and have been appearing since 2010. They were characterised as serogroup WII/III with 3 different susceptibility patterns corresponding to three different non-related PFGE profiles.

Conclusion This work confirms the reliability of the $\mathrm{Spx}$ for the presumptive identification of $\mathrm{Ng}$. Since several clinical laboratories in developing countries use only presumptive tests for $\mathrm{Ng}$ identification, is important to alert about these atypical isolates circulating in our country. It is important to be aware of using complementary tests for identification.

\section{P3.178 SYPHILIS IN PREGNANT WOMEN AND ELIMINATION OF CONGENITAL SYPHILIS IN BELARUS}

Pankratov Oleg. Belarusian Medical Academy of Postgraduate Education, Minsk - Republic of Belarus

\subsection{6/sextrans-2017-053264.413}

Introduction In Belarus the spread of syphilis (S) has been on the increase since 1988, and the incidence reached its peak in 1996 with 209.7 cases per 100000 inhabitants. During the following years the incidence has reduced with 7.2 cases in 2015. In the years 1996-2012 all together 5147 pregnant women infected with $S$ were registered in Belarus $(8.49 \%$ from the general number of the women with $S$ for this period - 60604 cases). The aim of the present study is to report on pregnant women infected with $\mathrm{C}$ and on elimination of congenital syphilis (CS) in Belarus.

Methods The study was retrospective. The data were obtained from the patients' files from the Dermatovenereological Dispensary in the city of Minsk, the state capital of Belarus, and regional Dermatovenereological Dispensaries of Belarus.

Results Primary S was diagnosed in $13.1 \%$ of pregnant women with S, secondary S $-30.2 \%$, latent early S $-55.8 \%$, latent late $\mathrm{S}-0.9 \%$. Disease has been revealed in I trimester of pregnancy in $57.1 \%$ of patients, II trimester $-23.1 \%$, III trimester - 17.2\%, after delivery - 2.6\%. 43.7\% of women have made abortion after disease revealing. Among women giving birth to children with CS primary $\mathrm{S}$ was not diagnosed, secondary S $-26.7 \%$, latent early $S-71.4 \%$, seroresistant $S$ - $1.9 \%$. Reinfection was registered in $8.5 \%$ of patients. There are 127 children with CS registered in 1994-2015 in Belarus in total. Early CS with symptoms has been revealed in 24 (18.9\%), early latent CS - in $103(81.1 \%)$ of children. Cases of late CS have not been registered. 2 cases of CS have been registered in 1994, 1995-12, 1996-13, 1997-14, 1998-23, 1999-18, 2000-6, 2001-8, 2002-7, 2003-8, 2004-5, 2005-1, 2006-4, 2007-1, 2008-2, 2009-1, 2010-2 cases. Last five years cases of CS have not been registered in Belarus.

Conclusion Belarus has adequate system of control of $S$ in pregnant women. In 2016 WHO certified the validation of elimination of CS as a public health problem in Belarus.

\section{P3.179 OBSTETRICAL ASSISTANCE ON HIV-POSITIVE PREGNANCIES MAY REDUCE VERTICAL TRANSMISSION}

MC Paschoini, FF Cunali Jr, PC Ferreira, LN Nogueira, NR Nogueira, MCS Scandiuzzi. Universidade Federal do Triangulo Mineiro, Uberaba - MG, Brazil

\subsection{6/sextrans-2017-053264.414}

Introduction Assess soropositivity of HIV infection among pregnant women overseen by Hospital de Clínicas from Universidade Federal do Triângulo Mineiro (HC-UFTM). Describe their demographical and epidemiological profile, obstetrical and newborn data.

Methods Survey through the records of pregnant women from HC-UFTM spanning from 2013 to 2016, followed by medical record review and administration of a structured questionnaire on epidemiological, obstetrical and neonatal aspects from HIVpositive pregnancies.

Results From January 2013 to November 2016, there were 69 births from HIV-positive women. On demography, the maternal age varied from 16 to 40 years, with 26.8 years average; the majority, $53.6 \%$ were in common-law marriages and 59.42 